\title{
Policies and Procedures for Sound-alike and Look-alike Medications
}

\author{
${ }^{1}$ Sheetal Singh, ${ }^{2}$ Angel R Singh
}

\section{ABSTRACT}

The sound-alike and look-alike (SALA) drug is one of the most common causes of medication error and is of fear globally. Thus, the probability for error due to confusing drug names is very high. The SALA drug names have become a significant challenge to pharmacists, nurses, patients, and doctors. With thousands of drugs currently on the market, the probability for error is significant. The purpose of this article is to formulate a policy that would help in minimizing medication errors arising with the use of SALA medications.

Keywords: Medication errors, Medication management, Patient safety, SALA medications

How to cite this article: Singh S, Singh AR. Policies and Procedures for Sound-alike and Look-alike Medications. Int J Res Foundation Hosp Healthc Adm 2017;5(1):15-20.

Source of support: Nil

Conflict of interest: None

\section{INTRODUCTION}

\section{Sound-alike and Lool-alike Medications}

Medication names that sound similar are identified as sound-alike medications and medication names in which packaging is visually similar to another product or medication are identified as look-alike medications. ${ }^{1}$ Sound-alike medications are those for which generic or trade names of the product or medication sound similar in the spoken or written words. Thus, they have a high potential for medication error.

Human factor concepts that are relevant to the medication use process are simplicity, standardization, differentiation, lack of duplication, and unambiguous communication. $^{2}$

\footnotetext{
${ }^{1}$ Project Consultant, ${ }^{2}$ Assistant Professor

${ }^{1}$ HITES, National Cancer Institute, All India Institute of Medical Sciences, Jhajjar, Haryana, India

${ }^{2}$ Department of Hospital Administration, National Cancer Institute, All India Institute of Medical Sciences, Jhajjar, Haryana India

Corresponding Author: Sheetal Singh, Project Consultant HITES, National Cancer Institute, All India Institute of Medical Sciences, Jhajjar, Haryana, India, e-mail: sheetalsingh2003@ hotmail.com
}

\section{NEED OF THE POLICY}

The SALA medications create serious problems in health care, leading to $29 \%$ of medication dispensing errors. Approximately $25 \%$ of errors reported to national medication error reporting programs is the aftermath of confusion with drug names that sound alike and look alike. ${ }^{3,4}$

\section{MATERIALS AND METHODS}

This was a descriptive cross-sectional study carried between March 2016 and March 2017. The study population included doctors, nursing personnel, pharmacists, and quality managers from tertiary care hospitals of both public and private hospitals of India. Checklist was made after an exhaustive review of literature, which was then improvised. Validation of the checklist was done by experts in quality assurance from the National Accreditation Board for Hospitals and Health care Providers-accredited private hospitals. Subsequently, interaction was done with study population against the backdrop of the checklist and policy was formulated.

\section{ANALYSIS AND RESULTS}

Two hundred people which included doctors, nurses, pharmacists, and quality managers of tertiary care public and private hospitals were approached for interaction against the backdrop of the check list. Total response rate was $60 \%$. A total of 25 responded out of 50 doctors approached, 35 nurses responded out of 50, 24 quality managers interacted out of 50 , and 36 pharmacists responded out of 50 approached. Policy was framed after incorporating inputs from responses received against the backdrop of the checklist.

\section{AIM}

The aim of the policy is to augment awareness among health care providers with regard to potential for medication errors due to SALA drugs, hence minimizing medication error.

\section{OBJECTIVES}

- To identify SALA medications, i.e., the drugs which have higher potential of medication error due to similarities in their nomenclature (generic/brand name) or their looks (in packaging). 
- To increase awareness among health care providers regarding SALA medications.

- To minimize medication errors arising with the use of SALA medications.

- To ensure ordering, storing, handling, and administration of SALA medications in a standardized manner.

\section{SCOPE}

- All areas of the health care facility where pharmacy is stored.

- All patient care areas of the hospital.

\section{RESPONSIBILITIES}

Pharmacist

Nursing staff

Clinicians

\section{POLICY}

\section{Strategies to Monitor SALA Drugs ${ }^{3,5-8}$}

- List of SALA medications to be prepared by the pharmacy of the health care facility. The list needs to be updated every 6 months.

- The list is to be prepared in two sets (Set 1 and Set 2) and drugs are stored in two separate racks/containers accordingly intended to avoid medication error due to close proximity of two SALA medications.

- To evade medication error, while dealing with SALA drugs, tall-man lettering should be practiced. In tallman lettering, part of a drug's name should be in upper case letters to distinguish SALA drugs from one another in order to avoid medication errors (e.g., predniSONE/predniSOLONE). ${ }^{9}$

- Generic names should also be included in the list of SALA drugs for reconfirming the selection of right medication.

- All nursing stations, treatment rooms, and other patient care area should have the list of SALA medications.

- Any query regarding SALA drugs should be answered by the pharmacy/clinicians at the earliest with correct information.

\section{How to Store SALA Drugs 3 ,5-8}

- The SALA medications should not be stocked up along with other medications, anywhere in the hospital.

- The SALA medications should be stored separately in two racks in the pharmacy store, Group I medications in one rack and group II medications in the other rack to avoid medication error.

- In the wards, emergency, and operation theater, the SALA drugs should be stored in separate drug bins or plastic boxes.
- In the pharmacy stores, the list of SALA medications should be hanging from both racks.

- In the ward/unit, the list should be pasted inside medication room, if required, on nursing station.

\section{Standardizing Warning Signage ${ }^{10}$}

Warning stickers pink in color and stating Sound-alike or Look-alike Medications need to be pasted on the storage racks in pharmacy.

In the ward/unit, emergency, or operation theater, cupboards storing the SALA drugs should have the pink colored SALA medication warning label/sticker outside the cupboard.

Inside the cupboard storing SALA drugs, the containers storing groups I and II SALA medications should have pink colored SALA warning sticker.

\section{How to Standardize Ordering/Prescription of SALA Drugs}

- While prescribing SALA medications, CAPITAL LETTERS should always be used and handwriting should be clear and legible.

- While ordering or prescribing SALA medications manually, both the brand and the generic name should be documented in the medication chart. ${ }^{10}$

- Formulary should be accessible to clinicians and staff nurses and facility should be provided for confirming

- Generic name from a brand name or

- Available brand names from a generic name

- Before documenting the prescription

- There should be readback confirmation of the drug name

- The generic composition of the name should be noted

\section{How to Dispense SALA Drugs}

Medication incidents linked to SALA drug names can pose serious harm to the patients. It is often difficult to detect the medication errors related to SALA drugs as the dispensed medication is presumed to have been the one that is prescribed for the patient. ${ }^{11}$

- At pharmacy or store while dispensing SALA medications, it should be doubly checked by another pharmacist.

- In wards, nursing staff should doubly check SALA medication before dispensing.

- In case of any doubt, it is mandatory that before dispensing SALA medications, the generic name of the drug to be dispensed should be confirmed from the doctors.

- The list of SALA medications should be made available in all the area from where drugs are being dispensed. 


\section{How to Administer SALA Drugs}

List of SALA medications should be available (pasted) in all medication rooms for cross reference.

Sound-alike and Look-alike drugs being high-risk medication, the patient should be monitored periodically, after administration of the drug, as this group of medications has high potential for medication error. ${ }^{12}$

List of look-alike medications is provided in Annexure 1. ${ }^{9}$

List of sound-alike medications is provided in Annexure 2. ${ }^{13}$

\section{Annexure 1}

\begin{tabular}{|c|c|c|c|}
\hline \multicolumn{4}{|l|}{ Look-alike drugs } \\
\hline Group I & & Group 2 & \\
\hline Brand name & Generic & Brand name & Generic \\
\hline Acivir $5 \mathrm{gm}$ Cream & Acyclovir & $\begin{array}{l}\text { Acivir Eye Ointment } 5 \mathrm{gm} \\
\text { Tube }\end{array}$ & Acyclovir \\
\hline Adaferin & Adapalene & Clindac A & Clindamycin \\
\hline Alex syrup & $\begin{array}{l}\text { Chlorpheniramine } \\
\text { maleate+Dextromethorphan }\end{array}$ & Alex (Sugar Free) & Chlorpheniramine maleate+Dextromethorphan \\
\hline $\begin{array}{l}\text { Alphagan P Eye } \\
\text { Drops }\end{array}$ & Brimonidine+purine derivatives & Alphagan Eye Drops & Brimonidine \\
\hline $\begin{array}{l}\text { Amantral } 100 \mathrm{mg} \\
\text { cap }\end{array}$ & Amantadine & Redotil cap & Racecadrotil \\
\hline Ambrodil Syrup & Ambroxol Hydrochloride & Ambrodil Plus Syrup & $\begin{array}{l}\text { Ambroxol Hydrochloride+Chlorpheniramine } \\
\text { maleate }\end{array}$ \\
\hline Ambrolite S Syrup & $\begin{array}{l}\text { Ambroxol Hydrochloride + } \\
\text { Salbutamol+Guaifenesin }\end{array}$ & Ambrolite D Syrup & $\begin{array}{l}\text { Cetirizine+Dextromethorphan+Pseudoephedr } \\
\text { ine } \mathrm{HCl}\end{array}$ \\
\hline Adriamycin INJ & Doxorubicin & Farmorubicin INJ & Farmorubicin \\
\hline Amclox & Ampicillin+Cloxacillin & Ceff 500 & Cephalexin \\
\hline Benzac AC & Benzoylperoxide & Premethrin & Premethrin \\
\hline Betadine Gargles & Povidine lodine & Betadine Solution & Povidine lodine \\
\hline Betnovate Cream & Betamethasone valerate & Betnovate N Cream & Betamethasone valerate+Neomycin \\
\hline $\begin{array}{l}\text { Bioclox Inj. } \\
500 \mathrm{mg}\end{array}$ & Cloxacillin & Biocillin Inj. 500 mg & Ampicillin \\
\hline Bricarex A Syrup & $\begin{array}{l}\text { Ambroxol Hydrochloride + } \\
\text { Terbutaline+Guaifenesin }\end{array}$ & Bricarex Syrup & Terbutaline \\
\hline Buscopan Tab & Hyoscine-N-butylbromide & Dulcolax Tab & Bisacodyl \\
\hline $\begin{array}{l}\text { Candid } \\
\text { Mouthpaint }\end{array}$ & Clotrimazole & Candid Topical Solution & Clotrimazole \\
\hline Cefmate $1 \mathrm{gm}$ & Cefoperazone+Sulbactum & Piptamate $4.5 \mathrm{gm}$ & Piperacillin+Tazobactum \\
\hline Ciplox Cream & Ciprofloxacillin & Ciplox D Cream & Ciprofloxacillin+Dexamethasone \\
\hline Clearz & Kozic Acid & Clearz Plus & Kozic Acid+ Vitamin C \\
\hline Clindac A & Clindamycin & Desowen & Desonide \\
\hline $\begin{array}{l}\text { Corex } 100 \mathrm{~mL} \\
\text { Syrup }\end{array}$ & $\begin{array}{l}\text { Codeine phosphate }+ \\
\text { Chlorpheniramine maleate+Alcohol }\end{array}$ & $\begin{array}{l}\text { Bron Corex } 100 \mathrm{~mL} \\
\text { Syrup }\end{array}$ & Bromhexine+Terbutaline+Guaiphenesin \\
\hline Coscopin & Noscapine & Coscopin Plus & $\begin{array}{l}\text { Noscapine+Sodium Citrate+Ammonium } \\
\text { Chloride+Chlorpheniramine Maleate }\end{array}$ \\
\hline Crocin & Paracetamol & Crocin DS & Paracetamol suspension \\
\hline Cutizone T cream & Mopmetasone Furoate+Terbinafine & Cutizone ointment & Mopmetasone Furoate \\
\hline Digene Tablets & $\begin{array}{l}\text { Dried aluminum Hydroxide+Mg Al } \\
\text { Silicate }+\mathrm{Mg}(\mathrm{OH}) 2\end{array}$ & Gelusil Tablets & $\begin{array}{l}\text { Aluminium hydroxide+magnesium } \\
\text { trisilicate+methyl polysiloxane }\end{array}$ \\
\hline Dipeptiven $50 \mathrm{~mL}$ & L-Alanine+L-Glutamine & Omegaven $10 \% 50 \mathrm{~mL}$ & EPA+DHA+Palmitic acid+Myristic acid \\
\hline Duoline & Salbutamol+|pratropium Bromide & Seroflo 500 rotacaps & Fluticasone \\
\hline Dutas $0.5 \mathrm{mg}$ & Dutasteride & Eldervit ZC & Vitamin B Complex \\
\hline Emoderm & $\begin{array}{l}\text { White Soft Paraffin Emollient cream } \\
\text { with Benzoic Acid }\end{array}$ & Emolene & Propylene glycol and Diazolidinyl Urea \\
\hline Emusone Cream & Clobetasone Propionate & Emusone M Cream & Clobetasone butyrate + Miconazole nitrate \\
\hline Facelin Gel & Clindamycin Phosphate & Facelin A Gel & Clindamycin+Adapalene \\
\hline
\end{tabular}

(Cont'd...) 
(Cont'd...)

\begin{tabular}{|c|c|c|c|}
\hline \multicolumn{2}{|l|}{ Group I } & \multicolumn{2}{|l|}{ Group 2} \\
\hline Brand name & Generic & Brand name & Generic \\
\hline Foracort Inhaler & Formoterol & Budecort inhaler & Budenoside \\
\hline Fucidine cream & Fusidic acid & Fucidine Ointment & Fusidic acid \\
\hline Fuucibet cream & Fusidic acid + betamethasone & Fucidin Cream & Fusidic acid \\
\hline Genteal Gel & Hydroxypropyl Methylcellulose & Genteal Eye Drops & $\begin{array}{l}\text { Hydroxypropyl Methylcellulose+boric acid + } \\
\mathrm{KCl}+\mathrm{NaCl}\end{array}$ \\
\hline Grillinctus Syrup & $\begin{array}{l}\text { Dextromethorphan }+ \text { guiphenesin }+ \\
\text { ammonium chloride }+ \\
\text { chlorpheniramine maleate }\end{array}$ & Grillinctus BM Syrup & Terbutaline+Bromhexine \\
\hline Halobet Cream & Halobetasol & Halobet S Cream & Halobetasol+Salicylic acid \\
\hline Ibugesic Syrup & Ibuprofen & Ibugesic Plus Syrup & Ibuprofen+Paracetamol \\
\hline Inj Magnex & Cefoperazone+Sulbactum & Inj Magnamycin & Cefoperazone sodium \\
\hline $\begin{array}{l}\text { Inj Solu Medrol } \\
40 \mathrm{mg}\end{array}$ & Methyl Prednisolone succinate & Inj Depo-Medrol 40 mg & Methyl Prednisolone acetate \\
\hline Inj Stancef 500 mg & Cefazolin Sodium & Inj Spectracef 500 mg & Cefditoren Pivoxil \\
\hline Inj Supacef 250 mg & Cefuroxime & Inj Fortum 250 mg & Ceftazidime \\
\hline Inj Taxim 500 & Cefotaxim & Inj Taximax 1500 & Cefotaxime Sodium +Sulbactum \\
\hline Inj Xylocaine & Lidocaine Hydrochloride & Inj Xylocard & Lidocaine Hydrochloride \\
\hline Intralipid $10 \%$ & Fat emulsion & Aminofen Infant $10 \%$ & Acetaminofen \\
\hline Liv 52 Syrup & Herbal Preparation (For appetite) & Mentat Syrup & Herbal Preparation (Antiparkinsonism) \\
\hline Meftal P & Mefenemic acid & Meftal Spas & Dicyclomine \\
\hline Nadoxin C Cream & $\begin{array}{l}\text { Clobetasol propionate }+ \\
\text { Nadifloxacin }\end{array}$ & Nadoxin Cream & Nadofloxacin \\
\hline Nucoxia -p Tablets & Etoricoxib + Paracetamol & Nucoxia Tablets & Etoricoxib \\
\hline Nizral & Ketoconazole & Surfaz SN & Clotrimozole+ Batamethasome+Neomycin \\
\hline Polybion Syrup & $\begin{array}{l}\text { Nicotinamide+pantothenyl alcohol } \\
\text { + vitamin B + vitamin B12 + vitamin } \\
\text { B2 + vitamin B6 }\end{array}$ & Polybion SF Syrup & Vitamin b Complex \\
\hline Riflux Forte & $\begin{array}{l}\text { Alginic acid + Dried aluminium } \\
\text { hydroxide + magnesium hydroxide } \\
\text { + sodium bicarbonate }\end{array}$ & Riflux & $\begin{array}{l}\text { Alginic acid+Dried aluminium } \\
\text { hydroxide+magnesium trisilicate + sodium } \\
\text { bicarbonate }\end{array}$ \\
\hline Rumalaya cream & Herbal preparation for pain & Pilex Ointment & Herbal preparation for pain (anti-hemorrhoid) \\
\hline Septillin Capsules & Herbal immunostimulant & Liv 52 Capsules & Herbal Preparation (For appetite) \\
\hline Septillin Syrup & Herbal immunostimulant & Koflet Syrup & Herbal preparation for cough \\
\hline Shelcal OS & Alphacalcidol & Shelcal $500 \mathrm{mg}$ & Calcium \\
\hline Syp Bacigyl & Norfloxacin & Syp Bacigyl - N & Metronidazole+norflocxacin \\
\hline Syp Dilosyn & Methdilazine & Exp Dilosyn & $\begin{array}{l}\text { Methdiazine hydrochloride+ Ammonium } \\
\text { Chloride +Sodium Citrate }\end{array}$ \\
\hline Syp Shelcal & Calcium+Vitamin d3 & Syp Shelcal M & $\begin{array}{l}\text { Boron+calcium carbonate+copper + magnesium } \\
\text { oxide + manganese + vitamin D3 + zinc }\end{array}$ \\
\hline $\begin{array}{l}\text { Taxim O } 200 \\
\text { Tablets }\end{array}$ & Cefixime & Taxim - O DT 50 & Cefixime Dispersible tablet \\
\hline T-Bact Ointment & Mupirocin & $\mathrm{T}$ - Bact cream & Mupirocin \\
\hline Telma H Tablets & Telmisartan + hydrochlorthiazide & Telma AM & Telmisartan + amlodepine besylate \\
\hline Tenovate Cream & Clobetasone Propionate & Tenovate G & Clobetasone butyrate + Gentamycin \\
\hline Tropacyl Plus & $\begin{array}{l}\text { Tropicamide + Phenylephrine } \\
\text { hydrochloride + Chlorbutol }\end{array}$ & Tears Plus & Ocular lubricant \\
\hline Venla XR Tablets & Venlafaxine XR & Roliten OD 2 mg Tablets & Tolterodine I-tartrate \\
\hline Visyneral Drops & Nutritional supplement & Visyneral Zn Drops & Nutritional supplement+zinc \\
\hline Zole ointment & Miconazole & Zole F Ointment & Miconazole+Flucinolone acetonide \\
\hline \multirow[t]{3}{*}{ Zytee } & $\begin{array}{l}\text { Choline salicylate + Benzalkonium } \\
\text { chloride }\end{array}$ & Surfaz SN & $\begin{array}{l}\text { Clotrimazole }+ \text { betamethasone dipropionate }+ \\
\text { neomycin sulphate }+ \text { tolnafatate }\end{array}$ \\
\hline & EPA - Eicosapentaenoic acid & & \\
\hline & DHA - Docosahexaenoic acid & & \\
\hline
\end{tabular}




\section{Annexure 2}

\begin{tabular}{|c|c|c|c|}
\hline \multicolumn{4}{|l|}{ Sound-alike drugs } \\
\hline \multicolumn{2}{|l|}{ Group 1} & \multicolumn{2}{|l|}{ Group 2} \\
\hline Brand name & Generic & Brand name & Generic \\
\hline aceRET 25 mg Cap & Acitretin & aceTEN 25 mg Tab & Captopril \\
\hline aceteC 25 mg Cap & Acitretin & aceteN 25 mg Tab & Captopril \\
\hline Acivir 200 mg DT Tab & Acyclovir & VALcivir $500 \mathrm{mg}$ Tab & Valacyclovir \\
\hline adENOCOR 2 mL Inj. & Adenosine & adRENOR 2 mL Inj. & Noradrenaline \\
\hline ALLegra 120 mg & Fexofenadine & EDegra $50 \mathrm{mg}$ & Viagra \\
\hline AMtas $10 \mathrm{mg}$ Tablets & Amlodepine & DUtas $0.5 \mathrm{mg}$ Cap & Dutasteride \\
\hline angiZEM $60 \mathrm{mg}$ Tablets & Diltiazem & angiSPAN $2.5 \mathrm{mg}$ Tab & Glyceryl trinitrate \\
\hline ATArax $10 \mathrm{mg}$ Tab & Hydroxyzine & ALPrax $0.5 \mathrm{mg}$ Tablets & Alprazolam \\
\hline ATORva 20 mg Tab & Atorvastatin & ARAva $20 \mathrm{mg}$ Tab & Leflunomide \\
\hline betaLOC $50 \mathrm{mg}$ Tab & Metoprolol & betaTROP $50 \mathrm{mg}$ Tab & Atenolol+nifedepin \\
\hline busCOPAN $10 \mathrm{mg}$ Tab & Hyoscine-N-butylbromide & busPIN 5 mg Tablets & Buspirone \\
\hline CellN 500 mg Tablets & Ascorbic acid & CeIEMIN 500 mL IV & Amino acids \\
\hline celemiN 500 IV & Parentral nutritional supplement & celemiX 500 IV & Amino acid+fat+sorbitol \\
\hline ciFRAN 500 mg Tablets & Ciprofloxacin & CiPLAR 10 mg Tablets & Propranolol \\
\hline ciFRAN 500 mg Tablets & Ciprofloxacin & septRAN & Sulphamethoxazole + Trimethoprin \\
\hline CIPLACtin Tablets & Cyproheptadine & CALAPtin $40 \mathrm{mg}$ Tablets & Verapamil \\
\hline clonlL $10 \mathrm{mg}$ Tablets & Clomipramine & clonOTRIL 0.5 mg Tablets & Clonazepam \\
\hline COLsprin CV $100 \mathrm{mg}$ Tablets & Aspirin low dose & ECOsprin AV 150 mg Cap & Aspirin+atorvastatin \\
\hline DEPSonil Tab & Imipramine & DAonil 5 mg Tab & Glibenclaimide \\
\hline dilCONTIN XL 100 mg Tab & Diltiazem & dilANTIN 25 mg Tab & Phenytoin \\
\hline dillGAN Tab & Meclizine & dilZEM $30 \mathrm{mg}$ tab & Diltiazem \\
\hline domTAC Tablets & Pantoprazole & domCET Tablets & Domperidone \\
\hline duOLIN Inhaler & Salbutamol+Ipratropium Bromide & duLANE $30 \mathrm{mg}$ Cap & Duloxetine \\
\hline ELOCon $5 \mathrm{~mL}$ Lotion & Mometasone furoate & EVALon cream $15 \mathrm{gm}$ Tube & Estriol \\
\hline EMESet $8 \mathrm{mg}$ Tablets & Ondansetron & DOMCet Tablets & Domperidone \\
\hline ENCorate & Sodium valproate & DICorate & Divalperoex sodium \\
\hline ENzar Forte Tablets & Amylase & VALzaar $40 \mathrm{mg}$ Tab & Valsartan \\
\hline EPidosin INJ & Valethamate & EPsolin INJ & Phenytoin \\
\hline FAMOCid $20 \mathrm{mg}$ Tablets & Famotidine & FERTOMid 25 mg Tab & Clomiphene \\
\hline FEStal N Tablets & $\begin{array}{l}\text { Pancreatin + bile constituents + } \\
\text { hemicellulase }\end{array}$ & MEFtal Forte Tablets & Mefenemic acid \\
\hline fungiZONE INJ & Amphotericin B (conventional) & fungiSOME INJ & Amphotericin B (Liposomal) \\
\hline Genteal $10 \mathrm{gm} \mathrm{Gel}$ & Hydroxypropyl methyl cellulose & Zentel $10 \mathrm{~mL}$ Syp Bottle & Albendazole \\
\hline GLImer 1 tab & Glimipride & GALAmer 4 mg Tab & Galantamine \\
\hline GLYnase 5 mg Tab & Glipizide & ZInase DP Tab & $\begin{array}{l}\text { Diclofenac potassium + } \\
\text { paracetamol + serratiopeptidase }\end{array}$ \\
\hline I kul 10 mL eye drop & Phenyl epherine & I gol 100 gm powder & Isabgol powder \\
\hline LIBrax 10 mg Tablets & $\begin{array}{l}\text { Chlordiazepoxide + Clidinium } \\
\text { bromide }\end{array}$ & LUBrex $10 \mathrm{~mL}$ eye drop & Sodium carboxyl methyl cellulose \\
\hline LARpose 2 mg Tablets & Lorazepam & CALMpose $5 \mathrm{mg}$ Tablets & Diazepam \\
\hline LOpez & Lorazepam & Topaz & Topiramate \\
\hline IorFAST 10 mg Tablets & Loratadine & IorVAS $2.5 \mathrm{mg}$ Tab & Indapamide \\
\hline Malirid $7.50 \mathrm{mg}$ Tablets & Primaquine & alreid Tablets & Cetrizine \\
\hline meftal FORTE Tablets & Mefenemic caid & meftal SPAS Tab & Dicyclomine \\
\hline metADAC 25 mg Inj. & Nandrolene decanoate & metOLAR 25 mg Tab & Metoprolol \\
\hline $\begin{array}{l}\text { MICROGest } 100 \text { mg capsule } \\
\text { nephroCAP Cap }\end{array}$ & $\begin{array}{l}\text { Progesterones + progestogens } \\
\text { Vitamin B }\end{array}$ & $\begin{array}{l}\text { MISOPRost } 100 \text { mg Tab } \\
\text { nephroSAVE Cap }\end{array}$ & $\begin{array}{l}\text { Misoprostol } \\
\mathrm{N} \text {-acetylcysteine +taurine }\end{array}$ \\
\hline NIMEgesic - OD Tablets & Nimesulide & IBUgesic 60 mL Syp Bottle & Ibuprofen \\
\hline novaCLOX 500 mg Capsules & Amoxycillin+Cloxacillin & novaMOX Tab & Amoxicillin \\
\hline NOVELOn Tablets & Progestogen+Estrogen & VOVERAn $50 \mathrm{mg}$ Tablets & Diclofenac Sodium \\
\hline novOLET MIXTARD 30HM & Human premixed insulin $(30 \%$ & novELON Tablets & Progestogen+Estrogen \\
\hline
\end{tabular}

(Cont'd...) 
(Cont'd...)

\begin{tabular}{|c|c|c|c|}
\hline Group 1 & & Group 2 & \\
\hline Brand name & Generic & Brand name & Generic \\
\hline numLO Tab & Amlodepine & numOL Tab & Diclofenac+Pracetamol \\
\hline OCid 10 mg Capsules & Omeprazole & ROXid 300 mg Tab & Roxithromycin \\
\hline Oflox 200 mg Tablets & Ofloxacin & NORflox $400 \mathrm{mg}$ Tablets & Norfloxacin \\
\hline PACIMOI 650 mg Tablets & Paracetamol & PRIMACAI Tab & Multivitamin+mineral \\
\hline POTrate 200 mL Syrup & Potassium Citrate & POTklor 200 mL Syp & Potassium Chloride \\
\hline PElox 400 mg Tablets & Pefloxacin & CIPlox 500 mg Tablets & Ciprofloxacin \\
\hline R CIFAx 200 mg Tablets & Rifaximin & R-CINEx 450 mg Tablets & Rifampicin + Isoniazid \\
\hline REXpar 200 mg Tablets & Sparfloxacin & RAClper 40 mg Tablets & Esomeprazole \\
\hline SAtrogyl 300 mg Tablets & Satranidazole & CYCLogyl $5 \mathrm{~mL}$ Eye drop & Cyclopentolate \\
\hline SAtrogyl $300 \mathrm{mg}$ Tablets & Satranidazole & MEtrogyl $400 \mathrm{mg}$ Tablets & Metronidazole \\
\hline seFDIN 300 mg Capsules & Cefdinir & seBIFIN 250 mg Tablets & Terbinafine \\
\hline SELOMax 50 mg Tab & Amlodepine+metoprolol & SOLIWax 10 mL Ear Drop & $\begin{array}{l}\text { Sodium 1,4-bis(2-ethylhexoxy)-1,4- } \\
\text { dioxobutane-2-sulfonate }\end{array}$ \\
\hline solET Tab & Sotalol & sollTEN 5 mg Tab & Solifenacin succinate \\
\hline SOLIWAx 10 mL Ear Drop & $\begin{array}{l}\text { Sodium 1,4-bis(2-ethylhexoxy)- } \\
\text { 1,4-dioxobutane-2-sulfonate }\end{array}$ & COLIMEx 10 mL Drop & Dicyclomine \\
\hline solONEX 300 mg Tab & Isoniazid & solIWAX 10 mL Ear Drop & $\begin{array}{l}\text { Sodium 1,4-bis(2-ethylhexoxy)-1,4- } \\
\text { dioxobutane-2-sulfonate }\end{array}$ \\
\hline sporANOX 100 mg Capsules & Itraconazole & sporIDEX AF 750 mg Tab & Cephalexin \\
\hline SYSCAn 150 mg Capsules & Fluconazole & SUSTEn 100 mg Cap & Progesterones+progestogens \\
\hline TOPCid 20 mg Tablets & Famotidine & ROXid $150 \mathrm{mg}$ Tablets & Roxithromycin \\
\hline TORLactone - 10 Tablets & Torsemide+Spironolactone & ALDactone $100 \mathrm{mg}$ Tablets & Spirinolactone \\
\hline TRENtal 400 mg Tablets & Pentoxifylline & TEGRItal 400 mg Tablets & Carbamazepine \\
\hline UROTone 25 mg Tab & Bethanechol Chloride & UTERone 100 mg Cap & Progesterones+progestogens \\
\hline valANCE 500 mg Tab & Divalproex & valENT 40 mg Cap & Valsartan \\
\hline xylocARD Inj $50 \mathrm{~mL}$ Vial & Lidocaine Hydrochloride & xylocAINE $2 \% 30 \mathrm{~mL}$ Inj. & Lidocaine Hydrochloride \\
\hline ZEMpred 8 mg Tab & Methylprednisolone & ACTOpred 500 mg Inj & $\begin{array}{l}\text { Methylprednisolone Sodium } \\
\text { Succinate }\end{array}$ \\
\hline ZINconia SYP & Zinc & ZINcovit SYP & Zinc +Multivitamins \\
\hline zeNTEL 400 mg Tab & Albendazole & zePTOL 100 mg Tab & Carbamazepine \\
\hline zinCOLAK Capsules & Zinc Sulfate Monohydrate & zinETAC $150 \mathrm{mg}$ Tab & Ranitidine \\
\hline Zocef $250 \mathrm{mg}$ Tablets & Cefuroxime & MONocef $250 \mathrm{mg} \operatorname{lnj}$ & Ceftriaxone \\
\hline zoCON 150 mg Tablets & Fluconazole & zoBONE Inj & Zoledronic acid \\
\hline zyrcoF Syrup & Cefadroxil + Ambroxal & zyrcoLD Tab & Cetirizine + Ambroxol \\
\hline ZYRop 2000 IU Inj & Erythropoietin & ZYRcof syrup & Cefadroxil + Ambroxal \\
\hline
\end{tabular}

\section{REFERENCES}

1. Ciociano N, Bagnasco L. Look alike/sound alike drugs: a literature review on causes and solutions. Int J Clin Pharm 2014 Apr;36(2):233-242.

2. Ismail S, Taqi A. Medical errors related to look-alike and sound-alike drug. Anaesth Pain Intensive Care 2013;17(2): 117-122.

3. Kenagy JW, Stein GC. Naming, labelling and packaging of pharmaceuticals. Am J Health Syst Pharm 2001 Nov;58(21):2033-2041.

4. Bates DW, Cullen D, Laird N, Peterson LA, Small SD, Servi D, Laffel G, Sweitzer BJ, Shea BF, Hallisey R, et al. Incidence of adverse drug events and potential adverse drug events: implications for prevention. J Am Med Assoc 1995 Jul;274(1): 29-34.

5. World Health Organization. WHO Collaborating Centre for patient safety solutions, look-alike sound-alike medication names. Patient safety solutions. Geneva: WHO; 2007.

6. Davis NM, Cohen MR, Teplitsky B. Look-alike and sound alike drug names: the problem and the solution. Hospital Pharmacy 1992;27:95-98, 102-105, 108-110.
7. McCoy LK. Look-alike, sound-alike drugs review: include look-alike packaging as an additional safety check. Jt Comm J Qual Patient Saf 2005 Jan;31(1):47-53.

8. Cohen MR, Anderson RW, Attilio RM, Green L, Muller RJ, Pruemer JM. Preventing medication errors in cancer chemotherapy. Am J Health Syst Pharm 1996 Apr;53(7):737-746.

9. Naunton M, Gardiner HR, Kyle G. Look-alike, sound-alike medication errors: a novel case concerning a slow-Na, slow-K prescribing error. Int Med Case Rep J 2015 Feb;8:51-53.

10. Ostini R, Roughead EE, Kirkpatrick CMJ, Monteith GR, TettSE. Quality use of medicines-medication safety issues in naming; look-alike, sound-alike medicine names. Int J Pharm Pract 2012;20(6):349-357.

11. Zaida R, Rukhsana P. Medication errors associated with look-alike/sound-alike drugs: a brief review. J Enam Med Col 2015 May;5(2):110-117.

12. Tuohy N, Paparella S. Look-alike and sound-alike drugs: errors just waiting to happen. J Emerg Nurs 2005 Dec;31(6):569-571.

13. Lambert BL, Lin SJ, Chang KY, Gandhi SK. Similarity as a risk factor in drug-name confusion errors: the look-alike [orthographic] and sound-alike [phonetic] model. Medical Care 1999 Dec;37(12):1214-1225. 